\title{
S-palmitoylation represents a novel mechanism regulating the mitochondrial targeting of BAX and initiation of apoptosis
}

\author{
M Fröhlich ${ }^{1}$, B Dejanovic ${ }^{1}$, H Kashkar ${ }^{2}$, G Schwarz ${ }^{\star, 1}$ and S Nussberger ${ }^{\star, 1,3}$
}

The intrinsic pathway of apoptotic cell death is mainly mediated by the BCL-2-associated $X$ (BAX) protein through permeabilization of the mitochondrial outer membrane (MOM) and the concomitant release of cytochrome $c$ into the cytosol. In healthy, non-apoptotic cells, BAX is predominantly localized in the cytosol and exhibits a dynamic shuttle cycle between the cytosol and the mitochondria. Thus, the initial association with mitochondria represents a critical regulatory step enabling BAX to insert into MOMs, promoting the release of cytochrome $c$ and ultimately resulting in apoptosis. However, the molecular mode of how BAX associates with MOMs and whether a cellular regulatory mechanism governs this process is poorly understood. Here we show that in both primary tissues and cultured cells, the association with MOMs and the proapoptotic action of BAX is controlled by its S-palmitoylation at Cys-126. A lack of BAX palmitoylation reduced BAX mitochondrial translocation, BAX oligomerization, caspase activity and apoptosis. Furthermore, ectopic expression of specific palmitoyl transferases in cultured healthy cells increases BAX S-palmitoylation and accelerates apoptosis, whereas malignant tumor cells show reduced BAX S-palmitoylation consistent with their reduced BAX-mediated proapoptotic activity. Our findings suggest that S-palmitoylation of BAX at Cys126 is a key regulatory process of BAX-mediated apoptosis.

Cell Death and Disease (2014) 5, e1057; doi:10.1038/cddis.2014.17; published online 13 February 2014

Subject Category: Cancer

Intrinsic apoptotic signaling converges on BCL-2-associated $\mathrm{X}$ protein (BAX), which drives the mitochondrial outer membrane permeabilization (MOMP) in the majority of cells. ${ }^{1-7}$ Failure in regulating this process can lead to distinct autoimmune diseases, ${ }^{8}$ cancer $^{9-11}$ and neurodegenerative disorders. ${ }^{12-14}$

During apoptosis BAX is inserted in the mitochondrial outer membrane (MOM) by a C-terminal tail anchor (TA). ${ }^{1}$ TA confers the association of cellular proteins mainly with the endoplasmic reticulum or mitochondria, where they are inserted into the lipid membrane via a single membrane span. ${ }^{15}$ Unlike the majority of TA proteins, including antiapoptotic Bcl-2 family members Bcl-2 and Bcl-xL and the proapoptotic $\mathrm{Bcl}-2$ family member BAK that are constitutively bound to their target membranes including mitochondrial and/or ER membranes, BAX in its monomeric inactive state predominantly appears in the cytosol of non-apoptotic cells. ${ }^{16}$ The solubility of BAX is effectively regulated by the reduced exposure of its hydrophobic $C$ terminus ${ }^{17}$ consistent with its predominantly cytosolic distribution in non-apoptotic cells, whereas only a minor fraction of BAX has been shown to be loosely attached to MOMs. ${ }^{18}$ Recent data conclusively showed that BAX exists in dynamic equilibrium between the cytosolic and mitochondrial compartment. ${ }^{18}$
Following apoptotic stimuli, BAX undergoes a conformational change to expose the reactive $\mathrm{BH} 3$ domain for dimer formation and to disengage the transmembrane anchor (TA) from the protein core, which in turn promotes its MOM insertion. ${ }^{17,19}$ In particular, the dimer formation via the $\mathrm{BH} 3$ domain has been shown to be facilitated in the presence of lipids, ${ }^{7}$ implying that the initial mitochondrial association of BAX provides a prerequisite for its subsequent activation by $\mathrm{BH} 3$ domain binding. However, the underlying molecular mechanism governing the dynamic and reversible equilibrium between cytosolic and mitochondrial BAX is largely unknown.

The structural and functional related proapoptotic BCL-2 protein BID exhibits striking similarities to BAX including the translocation to the MOM. In their inactive state both proteins show a predominantly cytosolic localization and accumulate at the mitochondria upon apoptosis induction. It was shown that BID acquires the affinity for MOM after its cleavage by caspase- 8 via a unique lipid modification, namely a post-translational myristoylation. ${ }^{20}$ As BAX unlike BID lacks a canonical consensus motif for myristoylation as well as the necessary N-terminal glycine, we asked whether S-palmitoylation at a cysteine residue could mediate the translocation of BAX to the outer mitochondrial membrane.

\footnotetext{
${ }^{1}$ Institute of Biochemistry, Department of Chemistry and Center for Molecular Medicine, Cologne University, Zülpicher Strasse 47, Cologne 50674, Germany; ${ }^{2}$ Institute for Medical Microbiology, Immunology and Hygiene and Center for Molecular Medicine, Cologne University, Goldenfels Strasse 19-21, Cologne 50935, Germany and ${ }^{3}$ Biophysics Department, Institute of Biology, University of Stuttgart, Pfaffenwaldring 57, Stuttgart 70550, Germany

*Corresponding author: G Schwarz, Institute of Biochemistry, Department of Chemistry and Center for Molecular Medicine, Cologne University, Zülpicher Strasse 47, Cologne 50674, Germany. Tel: +49 221470 6441; Fax: +49 221470 5092; E-mail: gschwarz@uni-koeln.de.

or S Nussberger, Biophysics Department, Institute of Biology, University of Stuttgart, Pfaffenwaldring 57, Stuttgart 70550, Germany. Tel: +49 71168565002 ; Fax: +49 7116856 5090; E-mail: nussberger@bio.uni-stuttgart.de

Keywords: apoptosis; BAX; DHHC protein family; mitochondria; S-palmitoylation; cancer

Abbreviations: MOM, mitochondrial outer membrane; MOMP, mitochondrial outer membrane permeabilization; TA, transmembrane anchor

Received 25.11.13; revised 23.12.13; accepted 02.1.14; Edited by G Raschellá
} 
Protein S-palmitoylation represents a unique lipid modification process mediated by members of the palmitoyltransferase family. S-palmitoylation serves as a reversible post-translational process mediating membrane targeting of various proteins. In particular, for proteins containing transmembrane segements, ${ }^{21-25} \mathrm{~S}$-palmitoylation is suggested to change the tilt of the transmembrane segments within the bilayer or alter its conformation, ${ }^{26}$ thereby promoting proteinprotein interaction and complex formation. ${ }^{25,27-29}$ On the basis of its ability to control the distribution and the function of a number of transmembrane- and membrane-associated proteins (e.g. associated with ER), here we asked whether S-palmitoylation of BAX could impact on the molecular switch regulating the dynamic and reversible equilibrium between cytosolic and mitochondrial BAX.

Our data show that a single cysteine residue in BAX is S-palmitoylated in tissue extracts and cell culture. Failure of BAX S-palmitoylation causes lack of BAX oligomerization, mitochondrial targeting and apoptosis. Congruently, enhanced S-palmitoylation of BAX by ectopic expression of distinct palmitoyl acyltransferases in cells increased sensitivity to apoptosis. By using malignant tumor cells derived from Hodgkin lymphoma $(\mathrm{HL})$ patients, we highlight the lack of BAX S-palmitoylation as an underlying molecular mechanism how these tumor cells resist apoptosis. ${ }^{30}$ This new mechanism may open up new avenues toward our understanding in the regulation of $B A X$ subcellular distribution and consequently its apoptotic activity.

\section{Results}

BAX is S-palmitoylated in tissue extracts and cell culture. To elucidate the molecular mechanism controlling the transit of BAX to and from mitochondria in response to apoptotic stimuli, we investigated lipid modification of BAX and used acyl biotin-exchange (ABE) chemistry. ${ }^{31}$ Probing equivalent amounts of protein with BAX-specific antibodies revealed endogenous $S$-palmitoylated $B A X$ protein in the brain, liver, lung, spleen, testis and kidney (Figure 1a), in human embryonic kidney 293 (HEK293) cells and in fibroblast-like green monkey kidney cells (Cos7) (Figure 1b). We further found palmitoylation of BAX in Sf9 insect cells following recombinant expression of human BAX (Figure 1b). Treatment of HEK293 cells with 2-bromopalmitate, a competitive inhibitor of protein acyltransferases, was found to reduce S-palmitoylation of endogenous BAX (Figure 1c). To further validate our findings, we performed ${ }^{3} \mathrm{H}$-palmitate incorporation experiments upon ectopic expression of BAX in Sf9 cells. Metabolic radiolabeling of BAX was shown by autoradiography (Figure 1d). These results demonstrate that BAX is post-translationally $\mathrm{S}$-palmitoylated, both in native tissues and cell culture.

To determine which residues of BAX are palmitoylated, we generated BAX variants in which Cys-62 and Cys-126, the only two cysteine residues and potential sites of S-palmitoylation, were replaced by serine. Variants were expressed with a C-terminal MYC epitope tag to allow a

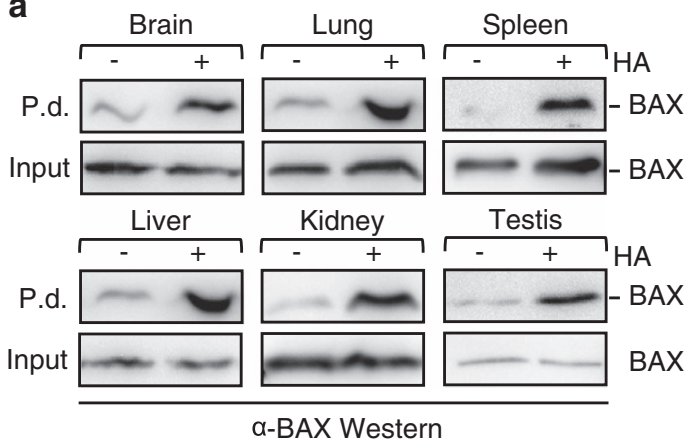

b
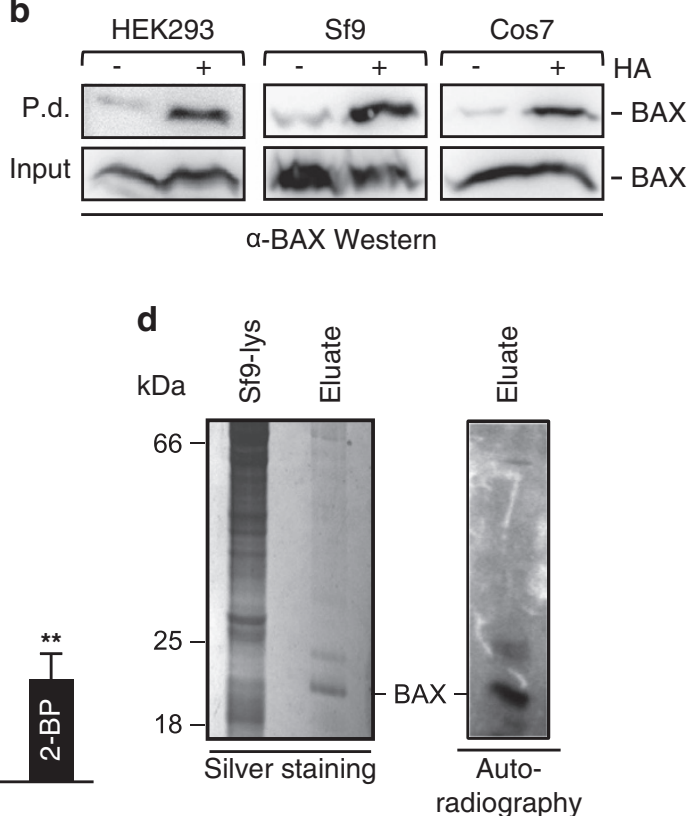

Figure 1 S-palmitoylation of BAX in mouse tissues and cultured cells. (a) Protein extracts from mouse tissues were tested for S-palmitoylated BAX following the ABE. Biotinylated proteins were purified, separated (by SDS-PAGE) and stained with anti-BAX antibodies ( $\alpha$-BAX). Biotinylation of BAX is prominent in protein extracts that were treated with hydroxylamine (HA); $n=2$. (b) S-Palmitoylation of BAX in HEK293 and Cos7 cells as well as SF9 cells expressing human BAX using the ABE; $n=2$. (c) Inhibition of protein acyltransferase for $16 \mathrm{~h}$ by 2 -bromopalmitate (2-BP) reduce BAX palmitoylation in HEK293 cells as shown by ABE. Quantification of relative amounts of palmitoylated $\mathrm{BAX}$ is shown. Error bars \pm S.E.M.; $n=6$; ${ }^{\star \star} P<0.01$. Input in panels (a-c) were $10 \%$ of total protein extract; $P$.d., pull down: protein bound to affinity resin. (d) Radiolabeling of BAX with ${ }^{3} \mathrm{H}$-palmitic acid $\left({ }^{3} \mathrm{H}-\mathrm{PA}\right)$. SF9 cells expressing for $48 \mathrm{~h}$ human His-tagged BAX were subsequently incubated with ${ }^{3} \mathrm{H}-\mathrm{PA}$ for $12 \mathrm{~h}$. BAX was isolated using Ni-affinity chromatography, run on SDS-PAGE and visualized either by silver staining or autoradiography 
a

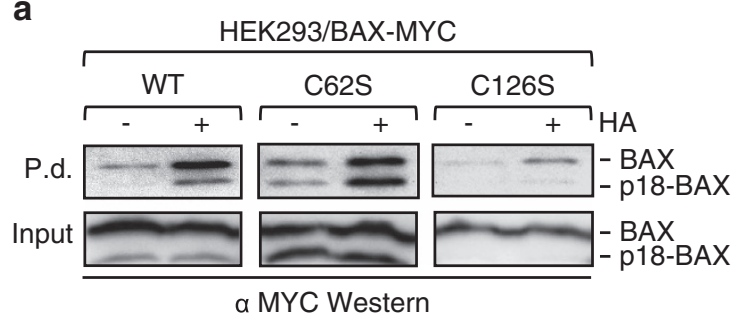

b

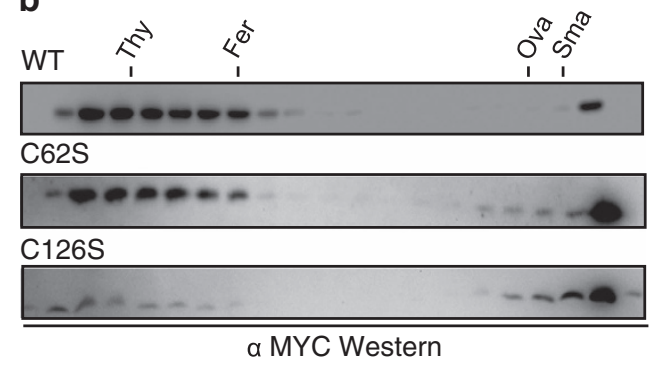

c

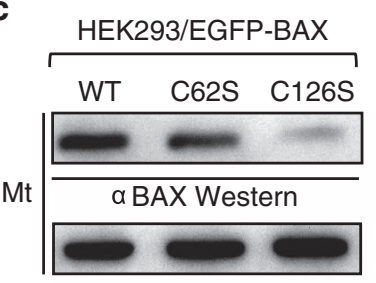

a VDAC Western

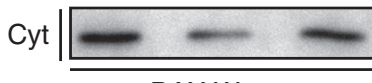

a BAX Western
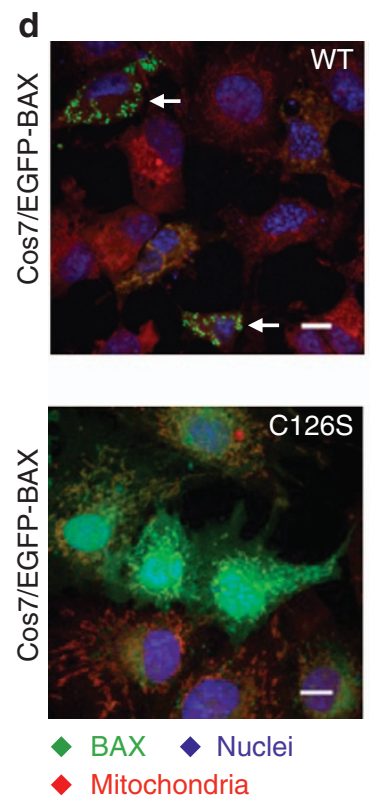

HEK293/BAX-MYC

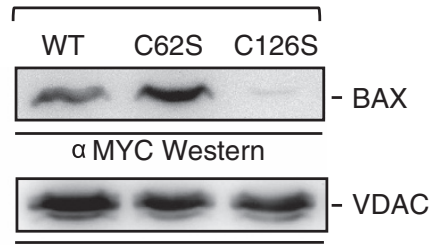

aVDAC Western

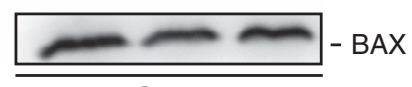

a MYC Western
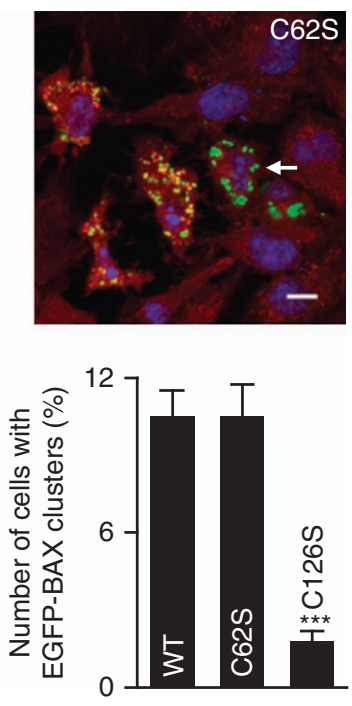

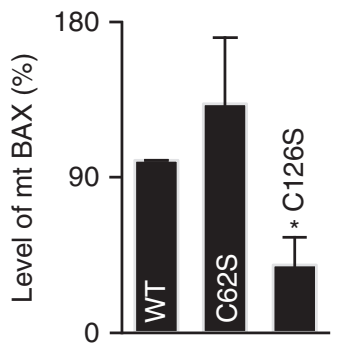

e
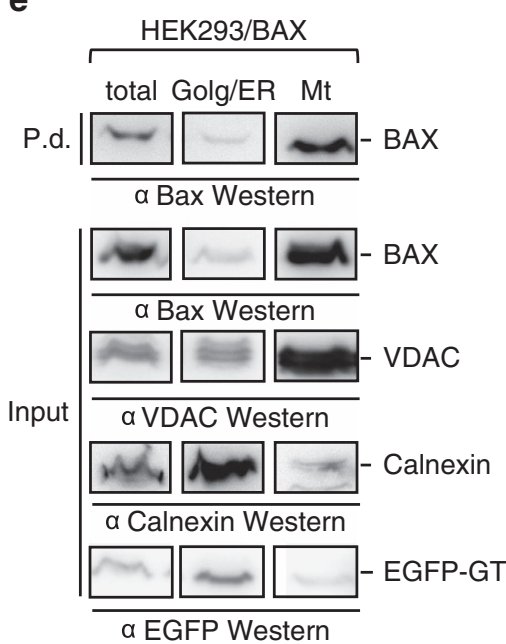

a EGFP Western

Figure 2 S-palmitoylation influences oligomerization and subcellular localization of BAX. (a) S-palmitoylation of MYC-tagged human BAX, BAX C62S or BAX C126S; $n=3$. Input, $10 \%$ of total protein extract; P.d., pull down, HA, hydroxylamine. (b) BAX oligomerization in HEK293 cells expressing MYC-tagged WT, C62S and C126S BAX analyzed by size-exclusion chromatography, followed by immunoblotting of fractions with anti-MYC antibodies. Thy, thyroglobulin (669 kDa); Fer, ferritin (440 kDa); Ova, ovalbumin (44 kDa); Sma, SMAC (25 kDa). (c) Mitochondria from HEK293 cells expressing C terminally MYC-tagged WT BAX, BAX C62S and BAX C126S, analyzed by immmunoblotting with anti-BAX or anti-MYC antibodies. VDAC, mitochondrial (Mt) marker. Bars (mean \pm S.E.M.; $n=4 ;{ }^{* *} P<0.01$ ) show quantification of relative amounts of mitochondrial BAX. Cyt, cytosol. (d) Cos7 cells transiently expressing WT and mutant BAX, N terminally fused with EGFP, were examined by microscopy for BAX clusters. Mitochondria were labeled with MitoTracker, nuclei with bisbenzimide. Number of cells with small punctate structures was quantified. Error bars \pm S.E.M.; $n=6$; ${ }^{* \star *} P<0.001$, Bars, $10 \mu \mathrm{m}$. (e) S-palmitoylated BAX copurifies with mitochondria isolated from HEK293 cells, transfected with Golgi marker (ECFP-GT). Proteins present in the Golgi/ER (Golg/ER) and mitochondrial fraction (Mt) were tested for palmitoylated BAX using ABE. VDAC, Calnexin and EGFP-GT were probed as markers for the respective fractions

unambiguous identification of exogenous BAX in cultured cells. Following transient expression in HEK293 cells, BAX was assayed for S-palmitoylation. Western blot analysis of protein extracts from cells expressing MYC-tagged wild-type (WT) and C62S BAX variants revealed two forms of BAX, the full-length $21 \mathrm{kDa}$ protein and a fragment with an approximate molecular mass of $18 \mathrm{kDa}$ (Figure 2a), both of which were $\mathrm{S}$-palmitoylated. By contrast, protein lysates from cells expressing the BAX C126S variant showed only a $21 \mathrm{kDa}$ band in the input and loss of the S-palmitoylation signal (Figure 2a). We conclude that S-palmitoylation of BAX occurs on residue Cys-126. The $18 \mathrm{kDa}$ fragment likely represents 
p18BAX, which is derived from full-length BAX via proteolytic cleavage following the initiation of apoptosis, ${ }^{32}$ a process that appeared to be absent or strongly reduced in BAX C126S.

S-palmitoylation influences oligomerization and subcellular localization of BAX. Given that the formation of higher-order oligomers is a prerequisite in BAX-mediated MOMP, ${ }^{33}$ we next investigated oligomer formation and the subcellular localization of BAX variants. Crude cell protein extracts of HEK293 cells expressing either WT, C62S or C126S BAX variants were clarified and separated by sizeexclusion chromatography (Figure 2b). Consistent with previous work, ${ }^{34}$ WT BAX eluted from the column in two separate fractions corresponding to $B A X$ monomers of $\sim 20 \mathrm{kDa}$ and $\mathrm{BAX}$ oligomers larger than $400 \mathrm{kDa}$. Although BAX C62S showed the same elution as WT BAX, BAX C126S was found primarily in the $20 \mathrm{kDa}$ fraction, indicating that the single $\mathrm{C} 126 \mathrm{~S}$ exchange reduced the propensity of BAX to form higher-order oligomers.

We next isolated mitochondria from HEK293 cells expressing either $\mathrm{N}$ terminally EGFP-tagged or $\mathrm{C}$ terminally MYCtagged WT, C62S or C126S BAX variants and detected them by western blot analysis using either anti-BAX or anti-MYC antibodies, respectively. In contrast to mitochondria from cells expressing WT or C62S BAX variants, which showed a strong BAX signal, only trace amounts of BAX C126S variant were found in the mitochondria (Figure 2c), whereas the mitochondrial marker VDAC was equally distributed. Cytosolic BAX was found with all three variants (Figure 2c). Consistent with the lack in forming high-order oligomers, BAX C126S variant was severely impaired in mitochondrial targeting. In contrast to our biochemical approach, we detected in addition to the cytosolic distribution an association of BAX C126S with mitochondria via fluorescence microscopy (Figure 2d), which was most likely due to the availability of endogenous BAX WT in Cos7 cells. However, this interaction of EGFPBAX C126S with the MOM did not result in a focal accumulation of EGFP-BAXC126S at mitochondrial sites, a hallmark for apoptotic cell death, ${ }^{35,36}$ as it was observed for EGFP-BAX WT and C62S (Figure 2d). In addition, the mitochondrial morphology in Cos7 cells expressing EGFPBAXC126S was not altered and the membrane potential remained unaffected, which was indicated by intact MitoTracker staining. Similar to previous work, ${ }^{35}$ Cos7 cells expressing EGFP-WT BAX or EGFP-BAX C62S showed punctate fluorescence staining in $\sim 10 \%$ of EGFP-BAXexpressing cells. In contrast, EGFP-BAX C126S showed mostly diffuse localization in the cytosol and a significantly reduced punctate staining ( $\sim 2 \%)$.

To address the question whether S-palmitoylated BAX specifically localizes to the mitochondria, we applied $A B E$ chemistry to identify BAX palmitoylation in different subcellular fractions of HEK293 cells. S-palmitoylation of endogenous BAX was high in fractions that were positive in the mitochondrial marker (VDAC) but low in fractions corresponding to the Golgi (1,4-galactosyltransferase-EGFP) and endoplasmic reticulum (Calnexin) (Figure 2e). In aggregate, all of our results suggest that S-palmitoylation of Cys 126 is crucial for BAX oligomerization and mitochondrial targeting.
Functional significance of S-palmitoylated BAX. High-level expression of BAX in mammalian cells induces mitochondrial cytochrome $c$ release, caspase activation and apoptotic body formation. ${ }^{1}$ On the basis of our observation that the BAX C126S variant is not processed to p18BAX (Figure 2a), which is believed to accelerate stress-induced apoptotic cell death, ${ }^{32}$ we determined the impact of Cys-126 in BAX-mediated apoptosis and analyzed apoptotic body formation and caspase-3 activity in Cos7 and HEK293 cells. The number of apoptotic Cos7 cells expressing BAX C126SMYC was significantly less than cells expressing WT or the C62S BAX variant (Figures $3 a$ and b). Accordingly, a reduced caspase-3 activity was found with BAX C126S as compared with WT and C62S BAX variant (Figure 3c). Similar results for the apoptotic body formation were obtained when EGFP-tagged variants were expressed in Cos7 cells (Figure $3 d$ ), indicating that S-palmitoylation of BAX at Cys-126 is important for the initiation of apoptosis. Surprisingly, we found that MYC-tagged BAX exhibited a higher potential to induce apoptosis compared with GFPBAX, which was mirrored by an increase in the total number of apoptotic bodies in the respective cell cultures.

S-palmitoylation of BAX is mediated by specific DHHC proteins. Protein S-palmitoylation is a frequent posttranslational modification in eukaroytic cells and mostly mediated by members of the DHHC (Asp-His-His-Cys) protein family. ${ }^{37}$ As palmitoylation occurs in a wide variety of sequence contexts within soluble and transmembrane proteins, S-palmitoylation is accommodated in mammalian cells by a large array of enzymes. ${ }^{37}$ To establish whether these proteins account for S-palmitoylation of BAX, we recombinantly expressed human WT BAX and each of the known 23 mouse DHHC proteins ${ }^{38}$ in HEK293 cells. After $16 \mathrm{~h}$ of coexpression, steady-state levels of BAX and $\mathrm{DHHC}$ proteins were determined and BAX palmitoylation was quantified using the $A B E$ assay (Figure $4 a$ and Supplementary Figure 1). BAX palmitoylation was compared with cells expressing BAX and GST as control. Twelve out of $23 \mathrm{DHHC}$ proteins appeared to stimulate S-palmitoylation of BAX. As expression levels of $\mathrm{DHHC}$ and $\mathrm{BAX}$ varied between repeating experiments and since the relative deviation between individual experiments $(n=7)$ strongly influenced the calculation of statistical significance by the $t$-test, certain $\mathrm{DHHC}$ proteins did not reach the threshold of $P<0.05$. Only coexpression of BAX and DHHC enzymes 3 , $7,11,12$ or 21 significantly stimulated S-palmitoylation of $\mathrm{BAX}$ as compared with control, suggesting that these $\mathrm{DHHC}$ enzymes are able to palmitoylate BAX.

Does S-palmitoylation of BAX in turn accelerate onset of apoptosis? To address this question, but to avoid stimulation of apoptosis through BAX overexpression, we expressed individual DHHC enzymes 3, 7, 11, 12 and 21 in HEK293 cells and treated the cells $24 \mathrm{~h}$ later with the antagonistic BCL-2 ligand $\mathrm{HA} 14-1^{39}$ to induce apoptosis. Caspase-3 activities were significantly elevated in cells expressing $\mathrm{DHHC}$ enzymes 3, 7, 11, 12 and 21 as compared with mocktransfected (GST expression) cells or cells expressing DHHC13, which did not stimulate S-palmitoylation of BAX (Figure $4 \mathrm{~b}$ ). In conclusion, transient expression of protein 
a

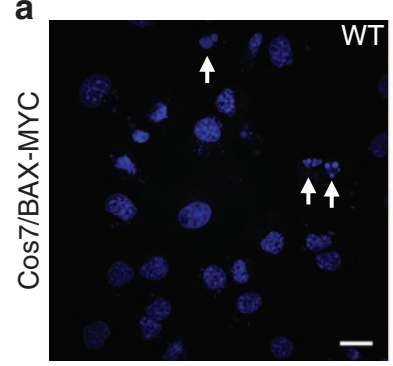

Nuclei / Apoptotic bodies

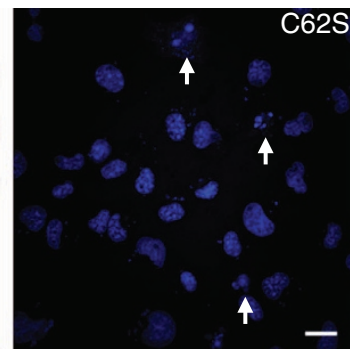

$+$

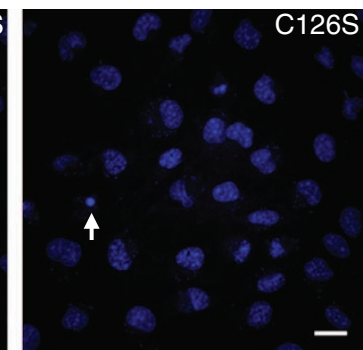

b

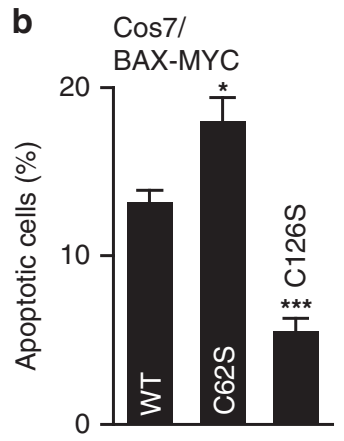

C

$$
\text { כ) }
$$
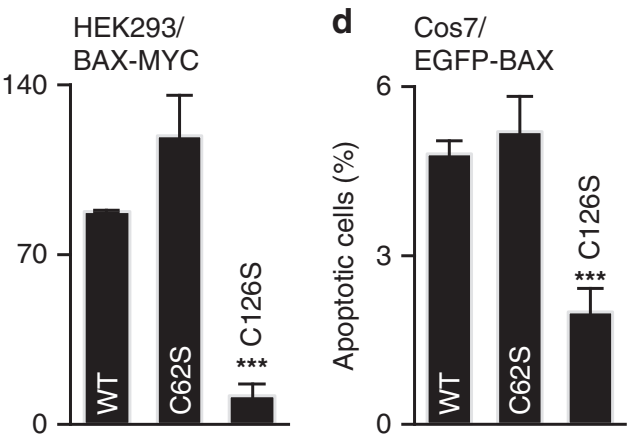

Figure 3 S-palmitoylation influences proapoptotic activity of BAX. (a) BAXC126S reduces apoptotic body formation. MYC-tagged BAX variants were expressed for $16 \mathrm{~h}$ in Cos7 cells. Bars, $20 \mu \mathrm{m}$. (b) Quantification of apoptotic Cos7 cells expressing BAX-MYC variants by assessing nuclear changes (apoptotic bodies) using the DNA-binding dye bisbenzimide. Error bars \pm S.E.M.; $n=6 \times 100$ cells; ${ }^{*} P<0.05$, ${ }^{* \star} P<0.001$. (c) BAX C126S reduces caspase-3 activity. Caspase-3 (cleavage of Ac-DEVD-AMC) activities were monitored in homogenates of HEK293 cells expressing WT, C62S and C126S BAX-MYC for $16 \mathrm{~h}$. Error bars \pm S.E.M.; $n=4$; ${ }^{* *} P<0.001$. (d) Quantification of apoptotic Cos7 cells expressing with WT, C62S and C126S EGFP-BAX for 16 h. Error bars \pm S.E.M.; $n=6 \times 500 ;{ }^{* * *} P<0.001$

acyltransferases mediating S-palmitoylation of BAX resulted in an increased HA14-1-stimulated apoptosis.

\section{S-palmitoylation of BAX is reduced in Hodgkin B cells.} Unlike in healthy individuals, B cells derived from patients with Hodgkin disease resist the majority of anticancer cytotoxic treatments based on the dysregulation of BAX activation. ${ }^{30,40}$ However, the underlying molecular mechanisms causing defective BAX action still remain elusive. To examine the impact of BAX S-palmitoylation on the observed lack of BAX activation in theses tumor cell lines, we examined the S-palmitoylation of BAX in protein extracts from healthy (e.g. L1309) versus four independent, previously described Hodgkin B-cell lines (L1236, L591, L481 and $\mathrm{KMH} 2){ }^{30}$ Although BAX expression levels were comparable in all cells ${ }^{30}$ and the palmitoylation machinery was found to be intact (Supplementary Figure 2), we observed markedly reduced palmitoylation of BAX in Hodgkin $B$ cells as compared with control B cells (Figure 4c). Palmitoylation of BAX thus clearly correlated with the previously reported lack of BAX activity in Hodgkin B-cell lines.

\section{Discussion}

The proapoptotic BCL-2 protein BAX is a key regulator in the intrinsic pathway of apoptosis, which critically influences the onset of apoptotic cell death at the level of MOM integrity. After induction of apoptosis, monomeric BAX is thought to translocate from the cytosol to the mitochondria, ${ }^{41}$ where it subsequently forms high-molecular-weight oligomers ${ }^{33,34}$ and induces the permeabilization of MOM. ${ }^{42}$ Although many studies elucidated distinct activation steps and discrete conformational changes of $\mathrm{BAX},{ }^{43-46}$ there is still a lack in understanding how BAX is redistributed to the mitochondria and which processes facilitate the affinity of BAX for MOM.

The idea of an unidirectional redistribution in response to an apoptotic stimulus is furthermore complicated by the observation that BAX can also be associated with MOMs in healthy, non-apoptotic cells. ${ }^{47,48}$ Recent studies additionally proved that in this case, BAX is not only localized in the cytosol or at mitochondria, but exhibit a dynamic translocation behavior between these two cellular compartments. ${ }^{6,18}$ Consequently, such a shuttling mechanism highlights the requirement for a dynamic and reversible biochemical process, which could act as a molecular switch on the activity of BAX and therefore account for the observed translocation cycles.

Our data highlight the S-palmitoylation of BAX as a possible novel post-translational regulatory circuit, which orchestrates $\mathrm{BAX} /$ mitochondria association and potentiates BAX proapoptotic activity. Although our data for the palmitoylation of BAX do not allow an unambiguous conclusion about the connection between the modification and the activity of the BAX protein, we are certain that the palmitoylation of BAX contributes to the reported translocation cycles between the cytosol and mitochondria. ${ }^{18}$

Notably, in a similar manner, a close relative of BAX, the proapoptotic BCL-2 protein BID, has been previously shown to be modified via $\mathrm{N}$-myristoylation, a process that intimately regulates its recruitment to $\mathrm{MOM} .{ }^{20}$ Taken together, these data underscore the critical role of lipid modifications as a 

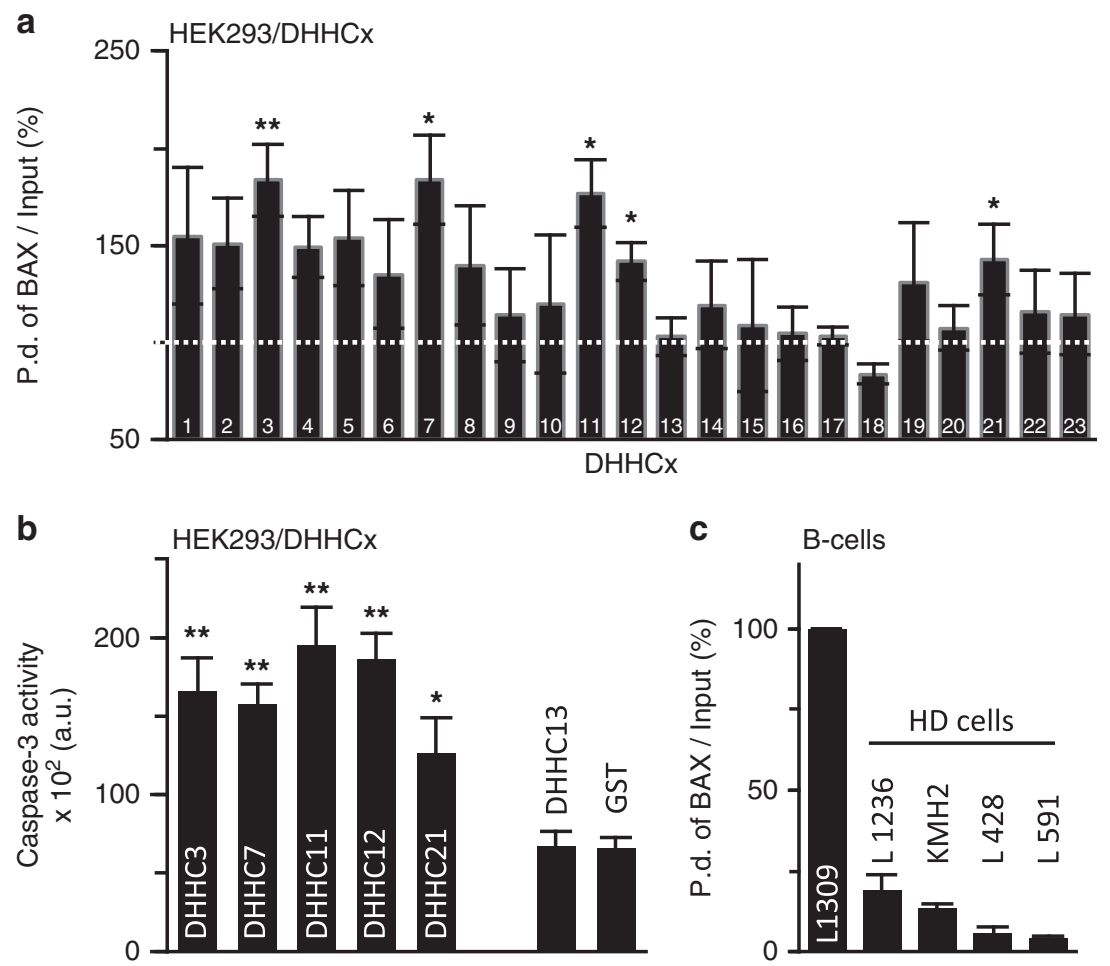

Figure 4 Stimulation and inhibition of BAX palmitoylation in healthy and malignant cells. (a) Stimulation of BAX palmitoylation. ABE palmitoylation assays were performed in HEK293 cells coexpressing BAX and DHHC1-23 for $16 \mathrm{~h}$. As a control, GST-expressing HEK293 cells were used (dotted line). Quantification of relative amounts of S-palmitoylated BAX is indicated. Error bars \pm S.E.M.; $n=3 ;{ }^{*} P<0.05 ;{ }^{*} P<0.01$. Input: $10 \%$ of total protein analyzed by ABE; P.d. pull down: protein bound to affinity resin. (b) Stimulation of caspase-3 activity. DHHC3, 7, 11, 12, 21 and 13 and GST were expressed in HEK293 cells and caspase-3 activity was monitored after $24 \mathrm{~h}$. Error bars \pm S.E.M.; $n=3$; ${ }^{*} P<0.05$, ${ }^{*} P<0.01$. (c) Protein extracts from control (L1309) and Hodgkin B-cell lines (L1236, L591, L481, KMH2, $n=2$ ) were tested for S-palmitoylated BAX following ABE

molecular switch governing the mitochondrial association and the proapoptotic action of BCL-2 proteins. BAX palmitoylation may thus be as important for cell death signaling as S-palmitoylation of CD95/Fas, which is required for receptor clustering in the plasma membrane and targeting to lipid membrane microdomains at an early step of apoptosis. ${ }^{49,50}$ At this stage, it is not yet clear whether palmitoylation of BAX is important for the dynamic regulation of membrane association through the regulated addition and removal of palmitoyl modifications as shown for proteins involved in intracellular signaling cascades, such as H/NRas, G-protein subunit $\mathrm{G}_{\mathrm{i} \alpha}$, PSD95 and GAP43, which can cycle between the plasma membrane and multiple endosomal membranes. ${ }^{51}$ If constant and fast cycles of palmitoylation and depalmitoylation are presumed, one could expect that there should be no palmitoylated BAX left at late time points after application of 2-BP. Nevertheless, the depalmitoylation of BAX is most likely influenced by the accessibility of its modified Cys-126 to palmitoyl protein thioesterases. Given that palmitoylated BAX inserts into the outer mitochondrial membrane, the modified Cys-126 is likely not accessible to the cytoplasmic environment and thus a depalmitoylation process would be unlikely to occur. Hence, the turnover of the modification would be significantly decreased and modified BAX detectable at late time points after application of 2-BP.

Previous data showed that BAX can be cleaved (p18) by cellular proteases including calpains during apoptotic and non-apoptotic cell death. ${ }^{52}$ The previous data concerning the proteolytic processing of BAX are so far not conclusively addressing whether and how p18 BAX interferes with cell death cascade. The elevated appearance of $p 18$ in the C62S variant of BAX in our experiments may be a result of an increased accessibility of BAX for the responsible protease, which is activated based on the cytotoxic activity of C62S BAX variant. Intriguingly, C126S BAX induces less cytotoxicity and the proteolytic processing of BAX in these cells is barely detectable.

S-palmitoylation of membrane proteins has been proposed (i) to occur most frequently in the proximity of their transmembrane segments to affect their conformation, (ii) to promote protein complex formation and (iii) to regulate their association with cholesterol- and sphingolipid-rich membrane domains. ${ }^{26}$ In line with these functions, we found that BAX is palmitoylated at a position (Cys-126), which is located at the C-terminal end of a transmembrane segment. Palmitoylation also appeared to promote protein complex formation. As mitochondria are known to maintain a specific sphingolipid milieu ${ }^{53}$ to promote BAX activation, ${ }^{43}$ it will be interesting to elucidate if palmitoylation targets BAX to sphingolipid membrane microdomains, as described for other palmitoylated membrane proteins. ${ }^{26}$

The site of BAX S-palmitoylation is located at the C-terminal end of the $\alpha 5$-helix being adjacent to the short positively charged loop Thr-127/Lys-128/Val129 connecting $\alpha 5$ and $\alpha 6 .{ }^{17,46}$ Both helices form a helical hairpin, which inserts into the outer mitochondrial membrane at an early 


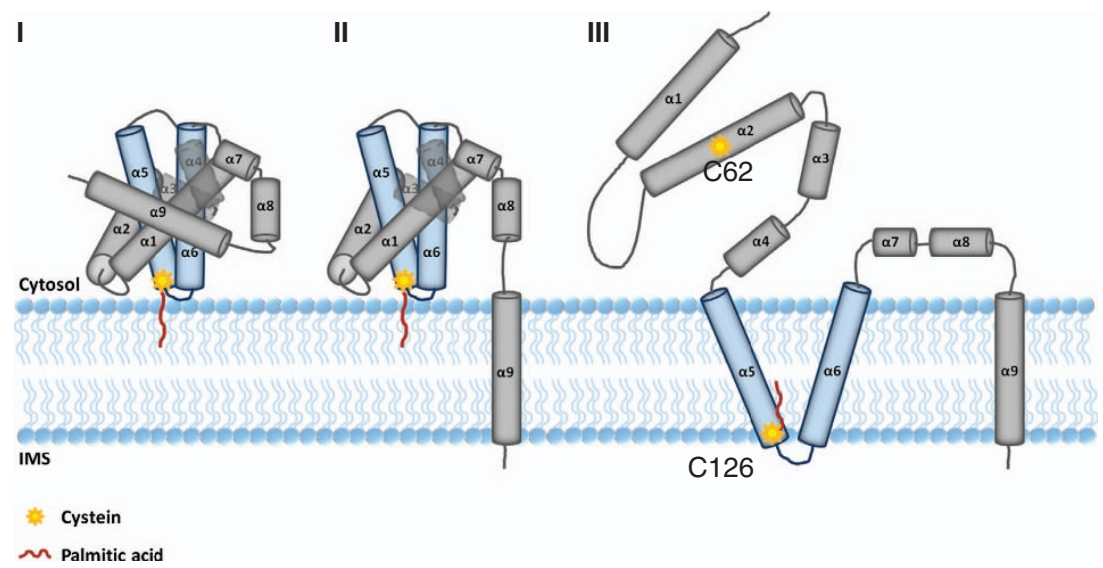

Figure 5 Possible modes of S-palmitoylation-mediated BAX insertion into the outer mitochondrial membrane. BAX mitochondrial membrane insertion represents a key step in apoptosis induction. Cys-126 of BAX becomes S-palmitoylated at the Golgi or at another endomembrane system by specific members of the DHHC protein acyltransferase family. The palmitoyl moiety, once captured by the MOM, orients BAX at the surface of the mitochondrium and facilitates integration of helices $\alpha 5$ and $\alpha 6$ (II and III) into the membrane. S-Palmitoylation of BAX may strongly favor formation of BAX dimers at the mitochondrial surface and subsequent assembly of higher order oligomers in the membrane. Both processes are required for the formation of a pore for the release of mitochondrial proapoptotic proteins into the cytosol such as apoptosis-inducing factor (AIF), Smac/DIABLO and cytochrome $c$. Both cysteines (Cys-62 and Cys-126) are highlighted in yellow. The palmitoyl moiety is marked in red. IMS, mitochondrial intermembrane space

stage of apoptosis. ${ }^{34,54}$ Although no common consensus sequences for S-palmitoylation has been identified yet, our results fit well with in silico prediction of palmitoylation sites. ${ }^{55}$ It is intriguing to speculate that the palmitoyl group at position Cys- 126 promotes membrane targeting of helices $\alpha 5$ and $\alpha 6$ to mitochondria and/or promotes their integration into the lipid bilayer (Figure 5). However, whether palmitoylation of Cys-126 induces tilting of the adjacent transmembrane domains in the manner as predicted for other membrane proteins $^{56}$ remains to be determined.

Using the established recombinant expression approach, ${ }^{38}$ we identified five potential BAX-modifying $\mathrm{DHHC}$ proteins (DHHC 3, 7, 11, 12 and 21), which were able to increase the relative palmitoylation levels of BAX. Interestingly, we found that upon expression of the respective $\mathrm{DHHC}$ proteins and therefore increased BAX palmitoylation, cells were highly sensitized toward apoptosis induction. In fact, after stimulation of apoptosis, the DHHC-expressing cells responded with a nearly twofold higher caspase- 3 activity compared with mock-transfected cells. Therefore, we could clearly demonstrate a functional correlation between the relative palmitoylation level of BAX and the initiation of apoptosis.

All identified DHHC proteins are known to be localized at the Golgi apparatus or the $\mathrm{ER},{ }^{57,58}$ which would implicate that BAX must access those organelles to be modified. Although it is well described that BAX can reside at both cellular compartments, ${ }^{5,59}$ the connection between the palmitoylation of $\mathrm{BAX}$ at the Golgi network or ER and the localization at the MOM still needs to be elucidated.

The physiological impact of BAX palmitoylation was validated by the analysis of $\mathrm{HL}$-derived $\mathrm{B}$ cells, which proved to be resistant to staurosporine-induced apoptosis. ${ }^{30}$ Notably, this resistance was based on the inability to convert BAX to its active state. Our analysis revealed that this insensitivity against staurosporine is accompanied by a tremendous reduction in the $\mathrm{BAX}$ palmitoylation levels compared with control B-cell line L1309.
Taken together, our data suggest that S-palmitoylation of BAX at Cys-126 represents a novel mechanism, which facilitates mitochondrial targeting of BAX, its oligomerization and BAX-mediated apoptosis. Consequently, the palmitoylation pathway of $\mathrm{BAX}$ identified in this work contributes to the control of MOM permeabilization. Finally, our finding provides a novel target to alter apoptosis in different cell types for the treatment of malignant, autoimmune and infectious diseases. ${ }^{60}$

\section{Materials and Methods}

Plasmids. All human BAX constructs (Supplementary Table 1) used for transfection of HEK293 and Cos7 cells were engineered by PCR and ligated into pcDNA3.1 (+)myc-His (Invitrogen, Darmstadt, Germany) and pEGFP-C2 (Clontech, Heidelberg, Germany) vectors. BAX constructs used for baculovirus infection of Sf9 cells were ligated into pVL1393 vector (BD Bioscience, Heidelberg, Germany). The plasmids encoding mouse DHHC PATs (pEFBOS/HA-DHHCX, $x=1-23$ ) were a gift from Masaki Fukata (Okazaki, Japan).

Expression constructs and mutagenesis. The mammalian expression plasmid pcDNA3.1 (+)myc-His/BAX encoding human BAX was generated by amplification of human $B A X$ gene with Hindll and Sful restriction sites and ligation into pcDNA3.1 $(+)$ (Invitrogen), resulting in a C-terminal MYC/(6 $\times)$ His-tagged protein. The amplification was carried out in two steps to add a Kozak sequence before the BAX coding region, to allow efficient protein expression of BAX. Residues Cys-62 and Cys-126 of the human $B A X$ gene were replaced with serine by fusion PCR using human $B A X$ as a template and the mutation primers C62S-fw/C62S-rev and C126Sfw/C126S-rev, respectively (Supplementary Table 1). The fusion PCR products were cloned with Hindll and Sful restriction sites into pcDNA3.1 + ). Pwo DNA polymerase in combination with Taq polymerase was used in each PCR step. Generation of recombinant baculovirus was carried out using baculovirus transfer vector pVL1393 (BD Bioscience). BAX was amplified by two separate PCRs using human $B A X$ as template and primers Sfg-fw/Sfg-rev (Supplementary Table 1) with an $3^{\prime}$ extension coding for a $(6 \times)$ His-tag. Amplified DNA fragments were then ligated into pVL1393 between the Smal and EcoRI restriction sites. Plasmids encoding GFP-tagged BAX were generated by cloning WT BAX, BAXC62S and BAXC126S into the Xhol/Sall sites of pEGFP-C2 (Clontech). To compensate a frameshift, an additional $C$ was added between the Xhol restriction site and the coding region of $B A X$. All plasmids that were amplified by PCR were verified by DNA sequencing.

Preparation of mouse organs. Organs were obtained from adult mice, washed with PBS, immediately frozen in liquid nitrogen, homogenized and stored at $-80^{\circ} \mathrm{C}$ until further use. 
Cell cultures and treatments. HEK293 and Cos7 cells were cultured at $37^{\circ} \mathrm{C}$ and $5 \% \mathrm{CO}_{2}$ in Dulbecco's modified Eagle's medium, $10 \%$ fetal bovine serum (FBS) and $2 \mathrm{mM}$ glutamine. B-cell lines (L1309, L1236, KMH2, L428 and L591) were cultured at $37^{\circ} \mathrm{C}$ and $5 \% \mathrm{CO}_{2}$ in RPMI 1640 medium, $10 \% \mathrm{FBS}, 2 \mathrm{mM}$ glutamine, $100 \mathrm{IU} / \mathrm{ml}$ penicillin and $100 \mu \mathrm{g} / \mathrm{ml}$ streptomycin. Sf9 cells were cultured as suspension cultures in spinner flasks at $27^{\circ} \mathrm{C}$ in modified Grace's insect medium (TNM-FH; AppliChem, Darmstadt, Germany), 10\% FBS, $50 \mu \mathrm{g} / \mathrm{ml}$ gentamicin and $2.5 \mu \mathrm{g} / \mathrm{ml}$ amphotericin B. For high-level expression of WT and mutant BAX, plasmids pcDNA3.1(+)myc-His/BAX, pcDNA3.1(+)myc-His/ BAXC62S or pcDNA3.1(+)myc-His/BAXC126S $\left(12.8 \mu \mathrm{g} \mathrm{cDNA} / 10 \mathrm{~cm}^{2}\right.$ plate; Supplementary Table 2) were transfected into HEK293 and Cos7 cells by using polyethylenimine (Sigma, Munich, Germany) and FuGENE 6 (Roche, Mannheim, Germany), respectively, for $16 \mathrm{~h}$. For transfection of Sf9 cells, a baculovirus was generated by co-transfection of pVL1393/BAX ( $1 \mu \mathrm{g} \mathrm{cDNA} / 25 \mathrm{~cm}^{2}$ flask) and BaculoGold DNA $(0.25 \mu \mathrm{g})$ using Baculo Gold Transfection Buffer A and B (BD Bioscience). Upon co-transfection, Sf9 cells synthesized BAX as well as viable BAX-coding virus particles. The latter were used to transfect Sf9 cells for large-scale production of BAX.

Acyl-biotinyl exchange assay. S-palmitoylation of protein was assessed by the acyl-biotinyl exchange assay as described. ${ }^{31}$ Briefly, total protein extracts $(2 \mathrm{mg} / \mathrm{ml})$ from mouse organs, cultured cells or purified mitochondria, Golgi and endoplasmic reticulum were incubated for $3 \mathrm{~h}$ at $40^{\circ} \mathrm{C}$ in solubilization buffer containing $4 \%$ SDS, 1.7\% Triton X-100, $5 \mathrm{mM}$ EDTA, $20 \mathrm{mM}$ methyl methanethiosulfonate (MMTS), $50 \mathrm{mM}$ Tris $(\mathrm{pH} 8.0) 1 \times$ protease inhibitor cocktail (Roche). Then, MMTS was removed from the protein extract by means of repeated chloroform-methanol precipitation. Protein pellets were solubilized in SDS buffer containing $4 \%$ SDS, $5 \mathrm{mM}$ EDTA, $50 \mathrm{mM}$ Tris $(\mathrm{pH} 8.0)$ and $1 \times$ protease inhibitor. After clarifying by centrifugation, supernatants were split into two and mixed with $0.2 \%$ Triton X-100, $0.4 \mathrm{mM} n$-[6-(biotinamido)hexyl]3'-(2'-pyridyldithio)-propionamide (HPDP-biotin; Thermo Scientific, Dreieich, Germany), $5 \mathrm{mM}$ EDTA and $1 \times$ protease inhibitor cocktail, respectively. One sample was supplemented with $1 \mathrm{M}$ hydroxylamine hydrochloride $(\mathrm{pH} 7.5)$. The other sample contained no hydroxylamine but $50 \mathrm{mM}$ Tris $(\mathrm{pH} 7.5)$. After $16 \mathrm{~h}$ of incubation at $4{ }^{\circ} \mathrm{C}$, protein of both samples was pelleted several times by chloroform-methanol precipitation and resuspended in $4 \%$ SDS, $5 \mathrm{mM}$ EDTA, $50 \mathrm{mM}$ Tris $(\mathrm{pH} 8.0)$ and $1 \times$ protease inhibitor cocktail. Biotinylated proteins were separated from non-biotinylated proteins by incubation with neutravidin agarose beads (Thermo Scientific). After $16 \mathrm{~h}$ of incubation at $4{ }^{\circ} \mathrm{C}$, the beads were washed four times with PBS supplemented with $0.2 \%$ Triton X-100 and $0.1 \%$ SDS, and bound proteins were eluted by incubation of beads with $2 \times$ SDS-PAGE loading buffer at $98^{\circ} \mathrm{C}$ for $5 \mathrm{~min}$. Finally, samples were submitted to SDS-PAGE and analyzed by immunoblotting with an anti-BAX antibody (BAX N-20; Santa Cruz Biotechnology, Heidelberg, Germany).

Metabolic palmitoylation of BAX with ${ }^{3} \mathrm{H}$-palmitic acid. Sf9 cells were infected with recombinant baculovirus containing the gene for $C$ terminally $(6 \times)$ His-tagged human BAX (pVL1393/BAX). Forty-eight hours after infection, Sf9 cells were incubated with $12 \mathrm{MBq} / \mathrm{ml}^{3} \mathrm{H}$-palmitic acid for $12 \mathrm{~h}$. Cells were lysed at $4{ }^{\circ} \mathrm{C}$ in $2 \% n$-octyl- $\beta$-D-glucoside, $300 \mathrm{mM} \mathrm{NaCl}, 40 \mathrm{mM}$ imidazole, $100 \mathrm{mM}$ Tris (pH 7.4) and EDTA-free protease inhibitor cocktail (Roche). After clarifying by centrifugation, the supernatant was bound to $\mathrm{Ni}^{2+}$-nitrilotriacetic resin (Qiagen, Hilden, Germany). Specifically bound material was eluted with $300 \mathrm{mM} \mathrm{NaCl}$, $500 \mathrm{mM}$ imidazole, $100 \mathrm{mM}$ Tris (pH 7.4) and submitted to non-reducing SDSPAGE. After fixation of the polyacrylamide gel in $40 \%$ methanol and $10 \%$ acetic acid, the gel was incubated for $1.5 \mathrm{~h}$ in enhancer solution (Perkin-Elmer, Rodgau, Germany), for $1 \mathrm{~h}$ in 5\% PEG 6000, dried under vacuum on 3MM filter paper (Whatman, Dassel, Germany) and analyzed by autoradiography.

Size-exclusion chromatography. HEK293 cells were harvested $12 \mathrm{~h}$ after transfection with pcDNA3.1(+)myc-His/BAX, pcDNA3.1(+)mycHis/BAXC62S or pcDNA3.1 $(+)$ myc-His/BAXC126S, washed with PBS and lysed in $2 \%$ CHAPS (3-[(3-cholamidopropyl)dimethylammonio]-1-propanesulfonate), $300 \mathrm{mM} \mathrm{NaCl}, 5 \mathrm{mM}$ EDTA, $100 \mathrm{mM}$ Tris (pH 8.0) and protease inhibitor cocktail. After clarifying by centrifugation, protein extracts $(5 \mathrm{mg})$ were loaded onto a Superdex 200 16/60 size-exclusion column (GE Healthcare, Freiburg, Germany) equilibrated with $300 \mathrm{mM} \mathrm{NaCl}, 1 \mathrm{mM} \mathrm{MgCl}, 100 \mathrm{mM}$ Tris (pH 8.0) and eluted at a flow rate of $1 \mathrm{ml} / \mathrm{min}$ at $4{ }^{\circ} \mathrm{C}$. Individual fractions were analyzed by SDS-PAGE and immunoblotting. Molecular masses were determined using UV spectra of chromatographs of thyroglobulin $(669 \mathrm{kDa})$, apoferritin $(443 \mathrm{kDa})$, ovalbumin $(44 \mathrm{kDa})$ and $\mathrm{Smac} / \mathrm{Diabolo}(25 \mathrm{kDa})$ as protein standards.

Caspase-3 activity measurements. HEK293 cells transfected with pcDNA3.1 (+)myc-His/BAX, pcDNA3.1 (+)myc-His/BAXC62S or pcDNA3.1(+) myc-His/BAXC126S were harvested $16 \mathrm{~h}$ after transfection and lysed by short sonication (3s) in ice-cold buffer containing $1 \%$ Triton $X-100,130 \mathrm{mM} \mathrm{NaCl}$, $10 \mathrm{mM}$ Na-pyrophosphate, $10 \mathrm{mM}$ Tris and $10 \mathrm{mM} \mathrm{NaH} \mathrm{PO}_{4} / \mathrm{Na}_{2} \mathrm{HPO}_{4}(\mathrm{pH} 7.5)$. Equivalent amounts of protein were added to a reaction buffer containing $20 \mu \mathrm{M}$ $\mathrm{N}$-acetyl-Asp-Glu-Val-Asp-7-amino-4-methylcoumarin (Ac-DEVD-AMC), $\quad 10 \%$ glycerol, $2 \mathrm{mM}$ dithiothreitol and $20 \mathrm{mM}$ HEPES pH 7.5). After incubation at $37^{\circ} \mathrm{C}$ for $2 \mathrm{~h}$, caspase- 3 activities were measured by monitoring the release of amino-4-methylcoumarin from Ac-DEVD-AMC using an Infinite 200 PRO mircoplate reader (Tecan, Crailsheim, Germany). Amino-4-methylcoumarin was excited at $380 \mathrm{~nm}$. Fluorescence emission was recorded at $460 \mathrm{~nm}$ with an integration time of $20 \mu \mathrm{s}$. The band widths for excitation and emission were 9 and $20 \mathrm{~nm}$, respectively.

Subcellular localization, confocal fluorescence microscopy and visual assessment of apoptotic cell death. Cos-7 cells were plated on collagen-coated glass coverslips and grown for $48 \mathrm{~h}$. Subsequently, the Cos7 cells were transfected with pEGFP-C2/BAX, pEGFP-C2/BAXC62S or pEGFP$\mathrm{C} 2 / B A X C 126 \mathrm{~S}$ and $16 \mathrm{~h}$ after transfection incubated with a mitochondrion-specific dye ( $75 \mathrm{nM}$ MitoTrackerRed CMXRos; Invitrogen) at $37^{\circ} \mathrm{C}$ for $1 \mathrm{~h}$. Cells were fixed with $4 \%$ paraformaldehyde in PBS for 20 min, quenched in $50 \mathrm{mM} \mathrm{NH}_{4} \mathrm{Cl}_{2}$ for $20 \mathrm{~min}$ and incubated with PBS containing DNA-specific dye $(1 \mu \mathrm{g} / \mathrm{ml}$ bisbenzimide; Applichem). After three additional PBS washing steps, the cover slips were washed with water and mounted with Mowiol containing 1,4-diazobicyclo-[2.2.2]-octane (Sigma). Images were recorded with an AZ-C2 ${ }^{+}$ confocal laser scanning microscope using a 60/1.4 Plan Apo oil immersion objective (Nikon, Düsseldorf, Germany). Fluorescent dyes/proteins were excited at a wavelength of $388 \mathrm{~nm}$ (bisbenzimide), $488 \mathrm{~nm}$ (MitoTrackerRed) and $568 \mathrm{~nm}$ (EGFP). Alternatively, HEK293 cells were transfected with pcDNA3.1 $(+)$ myc-His/ BAX, pcDNA3.1 (+)myc-His/BAXC62S and pcDNA3.1(+)myc-His/BAXC126S. Sixteen hours after transfection, mitochondria, Golgi and endoplasmic reticulum were isolated and analyzed by SDS-PAGE and immunoblotted with the indicated antibodies according to standard procedures. Apoptotic cell death was measured by counting the number of cells revealing apoptotic bodies.

Statistics. Statistical analyses were carried out using Student's t-test. $P$-values $<0.05$ were considered significant.

\section{Conflict of Interest}

The authors declare no conflict of interest.

Acknowledgements. We thank Masaki Fukata (Okazaki, Japan) for providing the plasmids expressing DHHC1-23 and Robin Ghosh (Stuttgart, Germany) for stimulating discussions. The work was supported by the Dual Career Program of the University of Cologne (SN) and the Fonds der Chemischen Industrie (GS).

\section{Author Contributions}

All authors read and approved the manuscript. MF designed, performed and analyzed the experiments; BD helped establishing assays; HK provided the knowledge to the analyses of Hodgkin B cells. GS and SN designed the experiments, analyzed the results and wrote the manuscript.

1. Youle RJ, Strasser A. The BCL-2 protein family: opposing activities that mediate cell death. Nat Rev Mol Cell Biol 2008; 9: 47-59.

2. Scorrano L, Oakes SA, Opferman JT, Cheng EH, Sorcinelli MD, Pozzan T et al. BAX and BAK regulation of endoplasmic reticulum $\mathrm{Ca}^{2+}$ : a control point for apoptosis. Science 2003; 300: 135-139.

3. Karbowski M, Norris KL, Cleland MM, Jeong SY, Youle RJ. Role of Bax and Bak in mitochondrial morphogenesis. Nature 2006; 443: 658-662.

4. de Brito OM, Scorrano L. An intimate liaison: spatial organization of the endoplasmic reticulum-mitochondria relationship. EMBO J 2010; 29: 2715-2723.

5. Dumitru R, Gama V, Fagan BM, Bower JJ, Swahari V, Pevny LH et al. Human embryonic stem cells have constitutively active Bax at the Golgi and are primed to undergo rapid apoptosis. Mol Cell 2012; 46: 573-583. 
6. Edlich F, Banerjee S, Suzuki M, Cleland MM, Arnoult D, Wang C et al. Bcl-x(L) retrotranslocates Bax from the mitochondria into the cytosol. Cell 2011; 145: 104-116.

7. Lovell JF, Billen LP, Bindner S, Shamas-Din A, Fradin C, Leber B et al. Membrane binding by tBid initiates an ordered series of events culminating in membrane permeabilization by Bax. Cell 2008; 135: 1074-1084

8. Mason KD, Lin A, Robb L, Josefsson EC, Henley KJ, Gray DH et al. Proapoptotic Bak and Bax guard against fatal systemic and organ-specific autoimmune disease. Proc Natl Acad Sci USA 2013; 110: 2599-2604.

9. Shibata MA, Liu ML, Knudson MC, Shibata E, Yoshidome K, Bandey T et al. Haploid loss of bax leads to accelerated mammary tumor development in C3(1)/SV40-TAg transgenic mice: reduction in protective apoptotic response at the preneoplastic stage. EMBO J 1999; 18: 2692-2701.

10. Rampino N, Yamamoto H, Ionov Y, Li Y, Sawai H, Reed JC et al. Somatic frameshif mutations in the BAX gene in colon cancers of the microsatellite mutator phenotype. Science 1997; 275: 967-969.

11. Beltran E, Fresquet V, Martinez-Useros J, Richter-Larrea JA, Sagardoy A, Sesma I et al. A cyclin-D1 interaction with BAX underlies its oncogenic role and potential as a therapeutic target in mantle cell lymphoma. Proc Natl Acad Sci USA 2011; 108: 12461-12466.

12. Chong MJ, Murray MR, Gosink EC, Russell HR, Srinivasan A, Kapsetaki M et al. Atm and Bax cooperate in ionizing radiation-induced apoptosis in the central nervous system. Proc Natl Acad Sci USA 2000; 97: 889-894.

13. Johnson BN, Berger AK, Cortese GP, Lavoie MJ. The ubiquitin E3 ligase parkin regulates the proapoptotic function of Bax. Proc Natl Acad Sci USA 2012; 109: 6283-6288.

14. Vila M, Jackson-Lewis V, Vukosavic S, Djaldetti R, Liberatore G, Offen D et al. Bax ablation prevents dopaminergic neurodegeneration in the 1-methyl-4-phenyl-1,2,3,6-tetrahydropyridine mouse model of Parkinson's disease. Proc Natl Acad Sci USA 2001; 98: 2837-2842.

15. Borgese N, Brambillasca S, Colombo S. How tails guide tail-anchored proteins to thei destinations. Curr Opin Cell Biol 2007; 19: 368-375

16. Hsu YT, Youle RJ. Bax in murine thymus is a soluble monomeric protein that displays differential detergent-induced conformations. J Biol Chem 1998; 273: 10777-10783.

17. Suzuki M, Youle RJ, Tjandra N. Structure of Bax: coregulation of dimer formation and intracellular localization. Cell 2000; 103: 645-654

18. Schellenberg B, Wang P, Keeble JA, Rodriguez-Enriquez R, Walker S, Owens TW et al. Bax exists in a dynamic equilibrium between the cytosol and mitochondria to control apoptotic priming. Mol Cell 2013; 49: 959-971.

19. Walensky LD, Gavathiotis E. BAX unleashed: the biochemical transformation of an inactive cytosolic monomer into a toxic mitochondrial pore. Trends Biochem Sci 2011; 36: 642-652.

20. Zha J, Weiler S, Oh KJ, Wei MC, Korsmeyer SJ. Posttranslational N-myristoylation of BID as a molecular switch for targeting mitochondria and apoptosis. Science 2000; 290: 1761-1765.

21. Alvarez E, Girones N, Davis RJ. Inhibition of the receptor-mediated endocytosis of diferric transferrin is associated with the covalent modification of the transferrin receptor with palmitic acid. J Biol Chem 1990; 265: 16644-16655

22. Schweizer A, Kornfeld S, Rohrer J. Cysteine34 of the cytoplasmic tail of the cationdependent mannose 6-phosphate receptor is reversibly palmitoylated and required for normal trafficking and lysosomal enzyme sorting. J Cell Biol 1996; 132: 577-584

23. Yik JH, Weigel PH. The position of cysteine relative to the transmembrane domain is critica for palmitoylation of $\mathrm{H} 1$, the major subunit of the human asialoglycoprotein receptor. J Bio Chem 2002; 277: 47305-47312

24. Veit M, Sollner TH, Rothman JE. Multiple palmitoylation of synaptotagmin and the t-SNARE SNAP-25. FEBS Lett 1996; 385: 119-123.

25. Abrami L, Leppla SH, van der Goot FG. Receptor palmitoylation and ubiquitination regulate anthrax toxin endocytosis. J Cell Biol 2006; 172: 309-320.

26. Blaskovic S, Blanc M, van der Goot FG. What does S-palmitoylation do to membrane proteins? FEBS J 2013; 280: 2766-2774.

27. Flannery AR, Czibener $C$, Andrews NW. Palmitoylation-dependent association with CD63 targets the $\mathrm{Ca}^{2+}$ sensor synaptotagmin VII to lysosomes. J Cell Biol 2010; 191: 599-613.

28. Valdez-Taubas J Pelham H Swf1-dependent palmitoylation of the SNARE TIg1 prevents its ubiquitination and degradation. EMBO J 2005; 24: 2524-2532.

29. Gubitosi-Klug RA, Mancuso DJ, Gross RW. The human Kv1.1 channel is palmitoylated, modulating voltage sensing: identification of a palmitoylation consensus sequence. Proc Natl Acad Sci USA 2005; 102: 5964-5968.

30. Kashkar H, Kronke M, Jurgensmeier JM. Defective Bax activation in Hodgkin B-cell lines confers resistance to staurosporine-induced apoptosis. Cell Death Differ 2002: 9: 750-757.

31. Wan J, Roth AF, Bailey AO, Davis NG. Palmitoylated proteins: purification and identification. Nat Protoc 2007; 2: 1573-1584.

32. Wood DE, Newcomb EW. Cleavage of Bax enhances its cell death function. Exp Cell Res 2000; 256: 375-382

33. Antonsson B, Montessuit S, Sanchez B, Martinou JC. Bax is present as a high molecula weight oligomer/complex in the mitochondrial membrane of apoptotic cells. J Biol Chem 2001; 276: 11615-11623

34. Annis MG, Soucie EL, Dlugosz PJ, Cruz-Aguado JA, Penn LZ, Leber B et al. Bax forms multispanning monomers that oligomerize to permeabilize membranes during apoptosis. EMBO J 2005; 24: 2096-2103.
35. Schinzel A, Kaufmann T, Schuler M, Martinalbo J, Grubb D, Borner C. Conformational control of Bax localization and apoptotic activity by Pro168. J Cell Biol 2004; 164: 1021-1032.

36. Karbowski M, Lee YJ, Gaume B, Jeong SY, Frank S, Nechushtan A et al. Spatial and temporal association of Bax with mitochondrial fission sites, Drp1, and Mfn2 during apoptosis. J Cell Biol 2002; 159: 931-938.

37. Greaves J, Chamberlain LH. DHHC palmitoyl transferases: substrate interactions and (patho)physiology. Trends Biochem Sci 2011; 36: 245-253.

38. Fukata M, Fukata Y, Adesnik H, Nicoll RA, Bredt DS. Identification of PSD-95 palmitoylating enzymes. Neuron 2004; 44: 987-996.

39. Wang JL, Liu D, Zhang ZJ, Shan S, Han X, Srinivasula SM et al. Structure-based discoveryof an organic compound that binds Bcl-2 protein and induces apoptosis of tumor cells. Proc Natl Acad Sci USA 2000; 97: 7124-7129.

40. Brinkmann K, Hombach A, Seeger JM, Wagner-Stippich D, Klubertz D, Kronke M et al. Second mitochondria-derived activator of caspase mimetic (SMAC) potentiates tumor susceptibility toward natural killer cell-mediated killing. Leuk Lymphoma 2013; e-pub ahead of print 26 June 2013

41. Wolter KG, Hsu YT, Smith CL, Nechushtan A, XiXG, Youle RJ. Movement of Bax from the cytosol to mitochondria during apoptosis. J Cell Biol 1997; 139: 1281-1292.

42. Jurgensmeier JM, Xie Z, Deveraux Q, Ellerby L, Bredesen D, Reed JC. Bax directly induces release of cytochrome $c$ from isolated mitochondria. Proc Natl Acad Sci USA 1998; 95: 4997-5002.

43. Kim H, Tu HC, Ren D, Takeuchi O, Jeffers JR, Zambetti GP et al. Stepwise activation of BAX and BAK by tBID, BIM, and PUMA initiates mitochondrial apoptosis. Mol Cell 2009; 36: 487-499.

44. Gavathiotis E, Reyna DE, Davis ML, Bird GH, Walensky LD. BH3-triggered structural reorganization drives the activation of proapoptotic BAX. Mol Cell 2010; 40: 481-492.

45. Dewson G, Ma S, Frederick P, Hockings C, Tan I, Kratina T et al. Bax dimerizes via a symmetric BH3:groove interface during apoptosis. Cell Death Differ 2012; 19: 661-670.

46. Czabotar PE, Westphal D, Dewson G, Ma S, Hockings C, Fairlie WD et al. Bax crystal structures reveal how $\mathrm{BH} 3$ domains activate $\mathrm{Bax}$ and nucleate its oligomerization to induce apoptosis. Cell 2013; 152: 519-531.

47. Goping IS, Gross A, Lavoie JN, Nguyen M, Jemmerson R, Roth K et al. Regulated targeting of BAX to mitochondria. J Cell Biol 1998; 143: 207-215.

48. Desagher S, Osen-Sand A, Nichols A, Eskes R, Montessuit S, Lauper S et al. Bid-induced conformational change of $\mathrm{Bax}$ is responsible for mitochondrial cytochrome $\mathrm{c}$ release during apoptosis. J Cell Biol 1999; 144: 891-901.

49. Feig C, Tchikov V, Schutze S, Peter ME. Palmitoylation of CD95 facilitates formation of SDSstable receptor aggregates that initiate apoptosis signaling. EMBO J 2007; 26: 221-231.

50. Chakrabandhu K, Herincs Z, Huault S, Dost B, Peng L, Conchonaud F et al. Palmitoylation is required for efficient Fas cell death signaling. EMBO J 2007; 26: 209-220.

51. Conibear E, Davis NG. Palmitoylation and depalmitoylation dynamics at a glance. J Cell Sci 2010; 123: 4007-4010.

52. Choi WS, Lee EH, Chung CW, Jung YK, Jin BK, Kim SU et al. Cleavage of Bax is mediated by caspase-dependent or -independent calpain activation in dopaminergic neuronal cells: protective role of Bcl-2. J Neurochem 2001; 77: 1531-1541.

53. Chipuk JE, McStay GP, Bharti A, Kuwana T, Clarke CJ, Siskind LJ et al. Sphingolipid metabolism cooperates with BAK and BAX to promote the mitochondrial pathway of apoptosis. Cell 2012; 148: 988-1000.

54. Schlesinger PH, Gross A, Yin XM, Yamamoto K, Saito M, Waksman G et al. Comparison of the ion channel characteristics of proapoptotic BAX and antiapoptotic BCL-2. Proc Nat Acad Sci USA 1997; 94: 11357-11362.

55. Ren J, Wen L, Gao X, Jin C, Xue Y, Yao X. CSS-Palm 2.0: an updated software for palmitoylation sites prediction. Protein Eng Des Select 2008; 21: 639-644.

56. Joseph M, Nagaraj R. Interaction of peptides corresponding to fatty acylation sites in proteins with model membranes. J Biol Chem 1995; 270: 16749-16755.

57. Ohno $Y$, Kihara A, Sano T, Igarashi $Y$. Intracellular localization and tissue-specific distribution of human and yeast DHHC cysteine-rich domain-containing proteins. Biochim Biophys Acta 2006; 1761: 474-483.

58. Korycka J, Lach A, Heger E, Boguslawska DM, Wolny M, Toporkiewicz M et al. Human DHHC proteins: a spotlight on the hidden player of palmitoylation. Europ. J Cell Biol 2012; 91: 107-117.

59. Nutt LK, Chandra J, Pataer A, Fang B, Roth JA, Swisher SG et al. Bax-mediated $\mathrm{Ca}^{2+}$ mobilization promotes cytochrome $\mathrm{c}$ release during apoptosis. J Biol Chem 2002; 277: 20301-20308.

60. Strasser A, Cory S, Adams JM. Deciphering the rules of programmed cell death to improve therapy of cancer and other diseases. EMBO J 2011; 30: 3667-3683.

(c) (1) $\Theta$ Cell Death and Disease is an open-access journal published by Nature Publishing Group. This work is licensed under a Creative Commons Attribution-NonCommercialNoDerivs 3.0 Unported License. To view a copy of this license, visit http://creativecommons.org/licenses/by-nc-nd/3.0/ 